Aufsatz

Sarah Kirchberger*

\title{
Eskalationsrisiken im Westpazifik - Inwieweit berühren diese die Interessen der NATO?
}

https://doi.org/10.1515/sirius-2021-4004

Zusammenfassung: Die NATO hat China erst seit wenigen Jahren auf dem Radarschirm und reagierte in diesem $\mathrm{Zu}$ sammenhang auf Herausforderungen, die sich durch eine zunehmende chinesische Präsenz in Europa und in den Global Commons sowie durch Chinas Zusammenarbeit mit Russland ergeben. Allerdings vernachlässigte sie bisher eine Betrachtung der strategischen Rolle, die sie vor dem Hintergrund des chinesisch-amerikanischen Sicherheitsdilemmas bei der Vermeidung eines Großmachtkonflikts spielen könnte. Auch findet bisher das Risiko einer strategischen Gleichzeitigkeit durch koordinierte chinesische und russische Provokationen in Ostasien und in Osteuropa zu wenig Beachtung. Die NATO sollte diese Risiken ernst nehmen, sich ihrer Einflussmöglichkeiten bewusst werden und beginnen, diese zu nutzen.

Schlüsselbegriffe: NATO, China, Russland, USA, Kriegsverhinderung

\begin{abstract}
NATO began looking systematically at China a few years ago as a reaction to challenges posed by China's increasing presence in Europe and within the global commons and as a result of Chinese-Russian cooperation. Nonetheless NATO has so far neglected to consider the strategic role it could play in averting a great-power conflict that might ensue from the Chinese-American security dilemma. Another area so far neglected concerns the risk of strategic simultaneity through coordinated Chinese and Russian provocations in East Asia and Europe. NATO should take these risks seriously; become aware of NATO's avenues of influence; and begin using them.
\end{abstract}

Keywords: NATO, China, Russia, USA, prevention of war

*Kontakt: Dr. Sarah Kirchberger, Institut für Sicherheitspolitik an der Universität Kiel (ISPK), Leiterin der Abteilung für Strategische Entwicklung in Asien-Pazifik, E-Mail: skirchberger@ispk.uni-kiel.de

\section{Einleitung}

Relativ spät, nämlich erst etwa Anfang 2018, hat die NATO begonnen, sich mit den Herausforderungen durch China intensiver zu befassen. Sie konzentrierte sich dabei auf Aspekte, die die Sicherheit Europas direkt berühren. Insbesondere einige eng wirtschaftlich mit China verflochtene europäische NATO-Mitgliedstaaten zögerten zuvor, die Herausforderungen durch China überhaupt auf die Tagesordnung zu setzen. Und auch nachdem dies geschehen war, betonte die NATO den rein defensiven Charakter ihrer Beschäftigung mit China. ${ }^{1}$

Entsprechend befasste sich auch die von Thomas de Maizière geleitete NATO-Reflexionsgruppe in ihrem Bericht NATO 2030 mit den unmittelbaren Herausforderungen der europäischen Sicherheit durch Chinas Präsenz in Europa. Sie konzentrierte sich auf (Cybersicherheits-) Risiken durch chinesische Investitionen in kritischen Infrastrukturen, die die Resilienz der NATO im Krisenfall untergraben könnten. Sie betonte die Sicherung der Lieferketten bei militärisch bedeutenden Technologien sowie die Gefahr durch chinesische Zulieferungen in Telekommunikationssysteme. Auch standen Chinas politische Einflussnahme auf Länder in Ost- und Südosteuropa im Mittelpunkt sowie die militärische Kooperation mit Russland in Europa, etwa im Rahmen gemeinsamer Übungen in europäischen Seegebieten. ${ }^{2}$ All dies ist richtig, dieser Blick spart jedoch die größere, strategische Perspektive weitgehend aus und ist zu kurz gedacht: er vernachlässigt das Sicherheitsdilemma zwischen den Vereinigten Staaten und China und die damit verbundenen Folgewirkungen für die Sicherheit Europas. In einem Beitrag für die Defense News mit dem Titel „NATO’s Focus on China is too

1 Remarks by NATO Secretary General Jens Stoltenberg on launching NATO2030 - Strengthening the Alliance in an increasingly competitive world, NATO, 8.6.2020, https://www.nato.int/cps/en/natohq/ opinions_176197.htm.

2 NATO 2030: United for a New Era. Analysis and Recommendations by the Reflection Group Appointed by the NATO Secretary General, NATO, 25.11.2020, https://www.nato.int/nato_static_fl2014/assets/ pdf/2020/12/pdf/201201-Reflection-Group-Final-Report-Uni.pdf. 
narrow" hat der US-Experte Hans Binnendijk auf diesen Punkt hingewiesen. Er schrieb, dass die NATO ihren Horizont gegenüber der chinesischen Herausforderung deutlich erweitern müsse und die strategischen Implikationen durch Chinas Versuche, das Gleichgewicht im Westpazifik zu verschieben, vor dem Hintergrund der Folgen für das Engagement der USA in Europa ernster nehmen sollte. Er sieht in diesem Zusammenhang vier Hauptaspekte: 1) Chinas Rolle als bestimmender Faktor für die sicherheitspolitische Ausrichtung der USA, die eine zunehmende Orientierung der Kapazitäten nach Ostasien erzwingt; 2) die strategische Koordination zwischen China und Russland, die zu einer Überlastung der NATO durch strategische Gleichzeitigkeit führen könnte; 3) Chinas Bemühungen zur Untergrabung der Freiheit in den Global Commons (was nicht nur Seewege, sondern auch Lufträume und sogar den Cyber- und Informationsraum betrifft) und die Implikationen chinesischer Projekte im Rahmen der Belt and Road Initiative (BRI) sowie im Weltraum und im Cyberspace; und 4) die realen Risiken einer militärischen Krise im Westpazifik, die Europa keineswegs unberührt lassen würde. $^{3}$

In Gesprächen tendieren insbesondere kontinentaleuropäische politische Akteure und Beobachter noch immer dazu, die direkten Folgen einer militärischen Krise im Westpazifik für die NATO zu unterschätzen. ${ }^{4}$ Zum einen wird das Risiko einer Eskalation ungeachtet der besorgniserregenden Veränderungen der Lage von vielen immer noch als verhältnismäßig gering eingestuft, sei es wegen der vermeintlichen militärischen Überlegenheit der USA oder aufgrund der Tatsache, dass sowohl die USA als auch China nuklear bewaffnet sind. Zum anderen wird häufig davon ausgegangen, dass es sich auch bei einem Worst Case-Szenario um einen lokal begrenzten und rein konventionell geführten Konflikt in Ost- oder Südostasien handeln würde, der das NATO-Kerngebiet als solches nicht direkt berühren und höchstens ein paar nachteilige Folgen für den Handel Europas mit Ostasien haben könnte. Solche Einschätzungen sind deutlich zu optimistisch und stehen in hartem Kontrast zur Sorge amerikanischer Experten und Operateure mit Erfahrung im Einsatzgebiet, die die Kriegswahrscheinlichkeit zwischen den USA und China mittelfristig sogar als hoch einschätzen

3 Hans Binnendijk: NATO's Focus on China is Too Narrow, Defense News, 3. April 2021, https://www.defensenews.com/opinion/ commentary/2021/04/03/natos-focus-on-china-is-too-narrow/.

4 Vgl. dazu den Meinungsbeitrag von Henrik Larsen: NATO Shouldn't Try to Do Too Much on China, The Diplomat, 16.7.2021, https:// thediplomat.com/2021/07/nato-shouldnt-try-to-do-too-much-onchina/. und die darauf verweisen, dass ein Konflikt mit einem peer competitor wie China vollkommen anders ablaufen würde als etwa der Irak-Krieg, nämlich äußerst verlustreich und nicht notwendigerweise erfolgreich. Wiederholt ergaben Konfliktsimulationen der US-Streitkräfte, dass selbst ein konventioneller Konflikt zu sehr hohen amerikanischen Verlusten führen würde und der Ausgang mehr als ungewiss wäre. ${ }^{5}$ Sicher wäre jedoch davon auszugehen, dass global genutzte Satellitenkonstellationen und kritische Cyberinfrastrukturen zu den ersten Opfern eines chinesischen Paralysierungsschlags zu Beginn von Kampfhandlungen gehören würden - mit direkten Folgen für alle NATO-Staaten, die solche kritischen Infrastrukturen nutzen. ${ }^{6}$ Die NATO stellte im Abschluss-Communiqué des Brüsseler Gipfels im Juni 2021 klar, dass bereits hybride Angriffe auf ein Mitglied, massive Cyberattacken sowie Angriffe auf Weltrauminfrastrukturen allein jeweils ausreichen können, um den Bündnisfall nach Artikel 5 auszulösen. ${ }^{7}$ Ein der chinesischen Militärdoktrin gemäß zu erwartender Paralysierungsschlag gegen kritische Systeme allein könnte demnach bereits ein möglicher Auslöser für den Bündnisfall sein.

Generell unterschätzen kontinentaleuropäische Kommentatoren auch das Risiko von chinesischen Angriffen auf amerikanische Territorien im Westpazifik wie Guam, die für die amerikanische Machtprojektion eine Schlüsselrolle spielen, und ebenso die Gefahr, dass eine Krise in Ostasien in Europa russische Ambitionen auf den Plan rufen könnte, die Ablenkung der USA und die Bindung ihrer Kräfte im Pazifik als günstige Gelegenheit für das Erreichen eigener Ziele im NATO-Gebiet auszunutzen. Erste Anzeichen für eine solche strategische Koordinie-

5 James Kitfield: “We're Going to Lose Fast.” U.S. Air Force Held a War Game that Started with a Chinese Biological Attack, Yahoo News, 10.3.2021, https://news.yahoo.com/were-going-to-lose-fast-us-airforce-held-a-war-game-that-started-with-a-chinese-biologicalattack-170003936.html; Brett Tingley: Joint Chiefs Seek A New Warfighting Paradigm After Devastating Losses In Classified Wargames, The Drive, July 27, 2021, https://www.thedrive.com/the-warzone/41712/joint-chiefs-seek-a-new-warfighting-paradigm-afterdevastating-losses-in-classified-wargames.

6 In der chinesischen Militärdoktrin spielt sogenannte „Systemzerstörungskriegsführung“ mit asymmetrischen Methoden, darunter Cyberattacken, ASAT, elektronische Kriegsführung, Informationsund psychologische Kriegsführung eine herausragende Rolle. Vgl. Engstrom 2018, iii, sowie aus chinesischer Sicht das 1999 im Original erschienene Buch der beiden VBA-Obersten Qiao Liang und Wang Xiangsui (Qiao/Wang 2015).

7 Brussels Summit Communiqué Issued by the Heads of State and Government participating in the meeting of the North Atlantic Council in Brussels 14 June 2021, https://www.nato.int/cps/en/natohq/ news_185000.htm, Abs. 31-33. 
rung von provokantem Verhalten beider Staaten häuften sich jedoch in den letzten Jahren. Im Frühjahr 2021 wurde die Möglichkeit einer solchen strategischen Gleichzeitigkeit zum ersten Mal deutlich fassbar, als der größte russische Truppenaufmarsch seit 2014 sich an der Grenze zur Ukraine positionierte und zugleich die von China provozierten Spannungen in der Taiwan-Straße massiv zunahmen. $^{8}$

Unter NATO-Mitgliedstaaten besteht dennoch immer noch weitgehend Uneinigkeit in der Lagebeurteilung, nicht nur über die Natur der chinesischen Herausforderung selbst, sondern auch über die Frage, ob und in welcher Form die NATO überhaupt mit der Abwehr von potenziellen Eskalationsrisiken im Westpazifik befasst sein sollte. Während das Vereinigte Königreich sich durch die Gründung des neuen Sicherheitsbündnisses AUKUS mit Australien und den USA sowie die Entscheidung zur dauerhaften Entsendung von Marineeinheiten in den Indopazifik inzwischen klar an der Seite der USA in der Großmachtkonkurrenz mit China positioniert hat, legen andere NATO-Mitglieder und insbesondere Deutschland und Frankreich Wert darauf, China entsprechend einer Einschätzung der EU weiterhin teils als „systemischen Rivalen“ und teils als „Wettbewerber“, teils jedoch auch als „Partner“ zu betrachten, und schrecken vor einer klareren Positionierung der NATO gegenüber China zurück. Globale Zukunftsprojekte wie der Kampf gegen den Klimawandel sind nach dieser Auffassung ein Grund, China trotz aller Schwierigkeiten weiterhin einzubinden, statt durch zu klare Positionierung auf Seiten der USA das Risiko einer Blockbildung einzugehen, die mit Entkoppelung von Wertschöpfungsketten, dem Rückbau von Handelsund Investitionsbeziehungen und einem allgemeinen Rückgang von wechselseitigem Austausch verbunden sein könnte, mit entsprechend hohen Kosten für einige Wirtschaftssektoren.

Vor diesem Hintergrund ist es gerade in Europa notwendig, die breiteren strategischen Zusammenhänge nach-

\footnotetext{
8 Vgl. Minnie Chan: China is going to hold live-fire navy drills in the Taiwan Strait in show of support for Russia over Syria, South China Morning Post, 12.4.2018, https://www.businessinsider.com/chinanavy-taiwan-strait-live-fire-drills-russia-syria-2018-4; Herman Pirchner, Jr./Alexander B. Gray: Taiwan's fate may be decided in Ukraine, The Hill, 4.6.2021, https://thehill.com/opinion/international/556826taiwans-fate-may-be-decided-in-ukraine; Ukraine, Taiwan, Middle East: The Worst-case Scenario For the Pentagon Takes Shape, MetaDefense, 12. April 2021, https://www.meta-defense.fr/en/2021/04/12/ ukraine-taiwan-middle-east-worst-case-scenario-for-the-pentagontakes-shape/; siehe auch Joachim Krause: Die Doppelkrise, die keiner wahrhaben will - dass China und Russland gleichzeitig militärisch eskalieren, ist kein Zufall, Neue Zürcher Zeitung, 24.4.2021.
}

zuvollziehen und eine nüchterne Aufarbeitung der Risiken vorzunehmen, die sich infolge des chinesisch-amerikanischen Sicherheitsdilemmas ergeben.

\section{Die NATO als Faktor im chinesisch-amerikanischen Sicherheitsdilemma}

Im Unterschied zur europäischen NATO-Perspektive auf China hatte China stets eine strategische Sicht auf die NATO. Diese wurde von jeher als ein Schlüsselinstrument amerikanischer Hegemonialmacht betrachtet, und entsprechend gründlich wurden sie und ihre Aktivitäten, insbesondere ihre Rolle in von den USA geführten Kriegen, analysiert. ${ }^{9}$ Chinesische Militärstrategen haben dadurch - im Vergleich zur traditionellen Nicht-Beschäftigung der NATO mit China - einen Vorsprung in der Einschätzung der Herausforderungen, die China durch die NATO an der Seite Amerikas erwachsen könnten, und haben sich entsprechende Gegenmittel überlegt.

Ähnlich wie Putins Russland ist Beijing langfristig an der Beendigung der amerikanischen Hegemonie und an der Schaffung einer multipolaren Weltordnung interessiert - diese wird euphemistisch als eine „Demokratisierung der internationalen Beziehungen“ bezeichnet. ${ }^{10}$ Das tatsächliche Verhalten sowohl Russlands als auch Chinas in ihrer jeweiligen Peripherie legt jedoch nahe, dass beider Ziel nicht eine „Demokratisierung“, sondern die Etablierung eines internationalen Systems konkurrierender Großmächte ist, die innerhalb ihrer jeweiligen Peripherie Einflusssphären kontrollieren und ihre Interessen gegenüber kleineren Staaten mit der Macht des Stärkeren rücksichtslos durchsetzen können. In Ostasien betrifft dies insbesondere Beijings erklärten Willen zur Einverleibung Taiwans.

Einer ungehinderten Durchsetzung chinesischer Interessen in Ost- und Südostasien steht vor allem die Rolle der USA als Sicherheitsgarant mehrerer demokratischer Nachbarstaaten Chinas im Weg. Peking verfolgt daher das Ziel, die USA aus dem Westpazifik heraus zu drängen und $\mathrm{zu}$ erreichen, dass ihre bilateralen Bündnisse und informellen Schutzgarantien insbesondere für die Staaten, die auf der China von Osten her einhegenden „Ersten Inselkette“ liegen (das sind von Nord nach Süd Südkorea, Japan, Taiwan und die Philippinen), beendet werden. So ist aus chinesischer Sicht mit einer „Denuklearisierung“

9 Vgl. hierzu Blasko 2009 und Dreyer 2011, siehe auch Roberts 2020. 10 Cooley/Nexon 2020, 81. 
der koreanischen Halbinsel nicht ein einseitiger Verzicht Nordkoreas auf Atomwaffen gemeint, sondern vielmehr der Abzug von US-Truppen aus Südkorea. ${ }^{11}$ Nur durch den Rückzug der USA könnte China in der Region langfristig selbst eine hegemoniale Rolle einnehmen, die seinem Selbstverständnis als Großmacht entspricht. Da die Demokratien Ostasiens jedoch ein Überlebensinteresse an der amerikanischen Schutzgarantie haben und die USA selbst zu einem Rückzug aus dem Westpazifik auch aus strategischem Eigeninteresse derzeit nicht bereit sind, besteht zwischen China und den USA ein Sicherheitsdilemma. Dieses verschärft sich zur Zeit durch Versuche Chinas, den Status quo durch hybride Methoden knapp unterhalb der Schwelle militärischer Gewalt zu verändern und zugleich durch massive Aufrüstung die militärische Balance zum Kippen zu bringen. ${ }^{12}$

Dieses Sicherheitsdilemma ist die treibende Kraft hinter der Ausrichtung der USA nach Asien, die unter Obama mit dem sogenannten Pivot to Asia begann, und auch hinter der jüngsten amerikanischen Entscheidung zum Rückzug aus Afghanistan. Das dem damaligen Vorsitzenden des NATO-Militärausschusses, Air Chief Marshal Sir Stuart Peach zufolge „schockierende“ Ausmaß der chinesischen Aufrüstung wiederum verstört nicht nur die USA, sondern hat einen Rüstungswettlauf in der gesamten Region einschließlich Australiens in Gang gebracht, dessen aktuell sichtbarster Ausdruck die Gründung neuer militärischer und militärtechnischer Abkommen wie QUAD und AUKUS ist. ${ }^{13}$

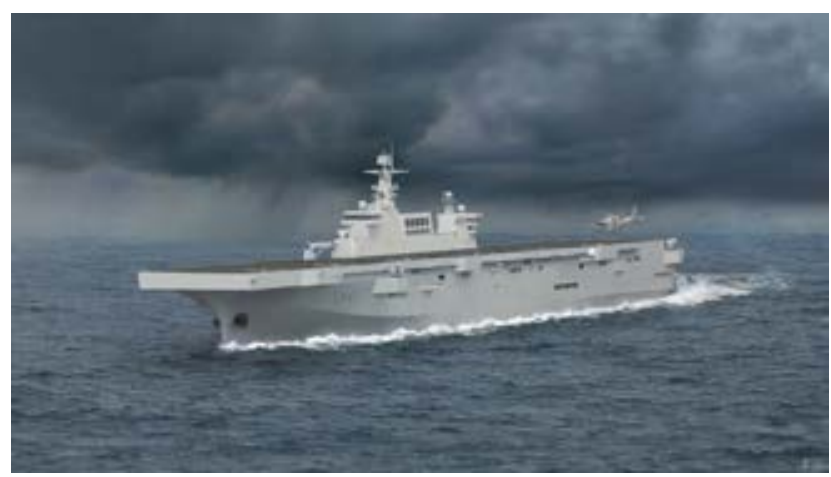

Chinesischer Hubschrauberträger des Typs 075

\section{Die Rolle Taiwans im chinesisch- amerikanischen Sicherheits- dilemma}

Insbesondere die Taiwan-Frage hat das Potenzial, vom lokalen Krisenherd zum großen Krieg zu eskalieren. Zwar gibt es keine formelle Beistandsverpflichtung der USA für Taiwan, aber die absichtlich vage gehaltene Selbstverpflichtung der USA im Taiwan Relations Act, die nach Abbruch der diplomatischen Beziehungen zu Taiwan 1979 die alte militärische Beistandspflicht der USA ersetzte, würde im Fall einer chinesischen Aggression mit hoher Wahrscheinlichkeit trotzdem greifen. Nicht zuletzt würde, wie Zbigniew Brzezinski betonte, durch amerikanische Untätigkeit im Angesicht einer chinesischen Aggression gegen Taiwan die gesamte Glaubwürdigkeit amerikanischer Bündnissysteme in ihren Grundfesten erschüttert und Amerikas Hegemonie dadurch beendet werden. ${ }^{14}$ Weiterhin hat die Insel Taiwan aufgrund ihrer geographischen Lage als „Eckpfeiler“ der Ersten Inselkette eine solch überragende Bedeutung für die Eindämmungsstrategie der USA gegenüber China, dass ihr Verlust kaum hinnehmbar wäre und das strategische Gleichgewicht nachhaltig zugunsten Chinas verschieben würde. Auf diese Tatsache wies am 15.9.2021 in ungewohnter Deutlichkeit der Außenminister Taiwans, Joseph Wu, hin, als er Taiwan auf einer Podiumsdiskussion mit amerikanischen Experten als „Sea Fortress“ - Seefestung - für die Eindämmung chinesischer Expansionsversuche bezeichnete. ${ }^{15}$ Die Einschätzung, dass die Kontrolle über die Insel Taiwan von zentraler Bedeutung für die maritime Expansionsstrategie Chinas wäre, wird von chinesischen Marinestrategen durchweg geteilt, da die Ostküste Taiwans direkten Zugang zum offenen Pazifik ermöglicht, was nicht zuletzt für die seegestützte nukleare Abschreckungsstrategie ein enormer Vorteil wäre. Denn bislang müssen chinesische Marineschiffe, um in den Pazifik zu gelangen, Durchlässe in der Ersten Inselkette passieren, die von Verbündeten der USA engmaschig überwacht werden. ${ }^{16}$ Und wie kürzlich der japanische Verteidigungsminister Kishi Nobuo klarstellte, berührt die Sicherheit Taiwans auch existenzielle Sorgen Japans, da eine chinesische Invasion Okinawas im Fall einer Taiwan-Krise aufgrund der räumlichen Nähe

14 Brzezinski 1997, 188-189.

15 Taiwan is ,sea fortress' against China, minister tells U.S. audience, Reuters, 15.9.2021, https://www.reuters.com/world/asia-pacific/ taiwan-is-sea-fortress-against-china-minister-tells-us-audience-202109-15/

16 Siehe z. B. Liu 2015, 180-83; 310, sowie Zhang 2014.
12 Holslag 2015, 16-20.

13 Helen Warrell/Michael Peel: Senior Nato Officer Warns of China's 'Shocking' Military Advances, Financial Times, 25.6.2021, https:// www.ft.com/content/8a0b3975-1938-4815-af3b-22b5d3e6aca4. 
aus rein taktischer Notwendigkeit heraus zu befürchten sei. Auch die umstrittenen, von Japan kontrollierten Senkaku-Inseln befinden sich in der Nähe Taiwans und wären dann gefährdet. ${ }^{17}$ Japan würde demnach vermutlich allein aus eigenem Sicherheitsinteresse heraus in einen militärischen Taiwan-Konflikt mit hineingezogen werden.

Schon diese wenigen Beispiele zeigen, dass die in Kontinentaleuropa immer noch häufig anzutreffende Vorstellung irrig ist, wonach es sich bei einem Krieg um Taiwan um einen klar abgegrenzten lokalen Konflikt handeln würde. Doch wie wahrscheinlich ist es, dass es zu einer konflikthaften Zuspitzung tatsächlich kommen könnte?

War die Kriegsandrohung Chinas gegenüber der „abtrünnigen Provinz“ Taiwan noch bis 2016 ein eher abstraktes Risiko, zeigen sich heute leider zahlreiche Anzeichen dafür, dass China sich aktiv auf ein Konfliktszenario vorbereitet, welches auch die Option einer Invasion einschließt. Dazu gehören massive Aufrüstungsmaßnahmen insbesondere bei Systemen, die bei einem Taiwan-Kriegsszenario zum Einsatz kommen würden. Aber auch der Abbruch jeglicher Kontaktkanäle zu den taiwanischen Behörden seit Mitte 2016 sowie die fortwährende Eskalationsrhetorik durch Drohungen und Schmähungen gehören hierzu. Weiterhin findet schon seit einiger Zeit eine hybride Destabilisierungsattacke gegen Taiwans Gesellschaft und politische Institutionen statt, bestehend aus Informationskriegsführung und Desinformationskampagnen, Cyberattacken sowie Unterwanderung und Subversion. Auffällig sind in jüngster Zeit die immer schärfer werdenden militärischen Drohgebärden. ${ }^{18}$

\section{Salami-Taktik und Anakonda-Methode}

Auf taktischer Ebene hat China in der vergangenen Dekade große Erfolge durch ein Vorgehen erzielt, das im Südchinesischen Meer als „Salami-Taktik“ und im Hinblick auf Taiwan als „Anakonda-Methode“ bezeichnet wird. Schritt für Schritt werden aggressive Maßnahmen knapp unterhalb der Schwelle direkter militärischer Aggression gegen einen schwächeren Gegenpart dazu genutzt, den Status quo immer weiter in die gewünschte Richtung zu verschieben. Im Südchinesischen Meer erfolgt dies durch das robuste Vorgehen staatlich organisierter, finanzierter,

17 Ya-chun Chang: MOFA welcomes Japan's interest in Taiwan Strait peace, Taiwan News, 9.9.2021, https://www.taiwannews.com.tw/en/ news/4283718.

18 Cole 2019. trainierter und koordinierter paramilitärischer Kräfte, die in Massenformationen gegen andere Anrainer wie die Philippinen oder Vietnam in Stellung gebracht und als „patriotische Fischer“ getarnt werden - „kleine blaue Männchen“ des Meeres. ${ }^{19}$ Mit ihrer Hilfe ist 2012 bereits die unblutige Eroberung des von den Philippinen kontrollierten Riffs Scarborough Shoal gelungen. In Bezug auf Taiwan dient dagegen eine Würgeschlangen- oder „Anakonda-Taktik“ dazu, mit Hilfe immer enger gezogener Umrundungsflüge durch militärische Jagd-, Bomber- und Aufklärungsflugzeuge sowie durch zunehmende Marineübungen in den Seegebieten rund um Taiwan die Bevölkerung einzuschüchtern und die taiwanischen Streitkräfte permanent herauszufordern und in ihrer Reaktionsfähigkeit letztlich zu überfordern..$^{20}$ Gemeinsam ist beiden Methoden, dass die kleineren Anrainer rein zahlenmäßig den chinesischen Kräften wenig entgegenzusetzen haben, und die hohe Frequenz der Übergriffe führt zu Ermüdung bei gleichzeitigem Nachlassen der internationalen Aufmerksamkeit. Dadurch erreicht China das Ziel, stetig taktische Vorteile zu erringen, eine riskante kriegerische Eskalation jedoch vorerst $\mathrm{zu}$ vermeiden.

Im Verlauf des 4. Oktober 2021 registrierte die taiwanische Luftwaffe mit insgesamt 56 Militärflugzeugen die bisher größte Zahl chinesischer Militärmaschinen, die jemals an einem Tag in die taiwanische Luftverteidigungszone eingedrungen sind. Es handelte sich um 4 Aufklärungsflugzeuge, 40 Kampfflugzeuge und 12 strategische Bomber in mehreren Wellen. Erst tags zuvor, am 3. Oktober, war die bis dahin höchste Zahl von 39 Militärflugzeugen an einem Tag registriert worden, einen Tag davor $38 .{ }^{21}$ Seit dem ersten derartigen Zwischenfall eines Eindringens chinesischer Militärflugzeuge in Taiwans Luftverteidigungszone im August 2016 sind Frequenz und Ausmaß solcher provokanten und der Einschüchterung Taiwans dienenden chinesischen Umrundungsflüge kontinuierlich angewachsen. Fanden sie in den ersten zwei Jahren seit Beginn etwa ein- bis zweimal pro Monat statt, zählte die taiwanische Luftwaffe im Jahr 2020 schon 380 Vorfälle, also im Schnitt mehr als einen pro Tag, und in den ersten neun Monaten des noch laufenden Jahres 2021 sogar bereits

19 Simon Tisdall: Little blue men: the maritime militias pushing China's claims, The Guardian, 16.5.2016.

20 Dreyer 2018.

21 Die Streitkräfte der Republik China auf Taiwan veröffentlichen auf ihrer offiziellen Website regelmäßig Daten zu den beobachteten Verletzungen der taiwanischen Luftverteidigungszone (ADIZ). Diese können jeweils tagesaktuell unter dem Link https://www.mnd.gov. tw/PublishTable.aspx?Types=即時軍事動態\&title=國防消息 abgerufen werden. 
mehr als 500 Vorfälle. Die immer größeren Geschwader bestehen überwiegend aus Kampfflugzeugen sowie strategischen Bombern und Aufklärungsflugzeugen. ${ }^{22}$ Die taiwanische Luftwaffe ist inzwischen nicht mehr in der Lage, bei jedem Eindringen chinesischer Militärflugzeuge in die eigene Luftverteidigungszone Abfangjäger aufsteigen $\mathrm{zu}$ lassen, und die Überlastung ihrer Luftabwehr hat bereits zu mehreren tödlichen Unfällen und Verlusten von schwer zu ersetzenden Piloten und Flugzeugen geführt, während chinesische Militärs durch solche Operationen jedes Mal wertvolle Informationen über das Terrain und die gegnerischen Abwehrfähigkeiten erwerben. Zwar haben diese Drohgebärden eindeutig eine symbolische und innenpolitische Komponente, aber es wäre ein Fehler, sie nicht auch als Teil einer Vorbereitung auf ein Konfliktszenario zu begreifen. Wie Ian Easton in seiner 2017 erschienenen Studie The Taiwan Invasion Threat basierend auf dem Studium von Dokumenten der chinesischen Volksbefreiungsarmee (VBA) zur Invasionsplanung darlegt, stellt die Eroberung Taiwans das zentrale Ziel aller chinesischen Militärplanung dar: „It is something that has been indoctrinated and encoded into the minds of all top-level officers. This offensive operation shapes their lives and institutions. It defines them and gives their military service purpose and meaning. For them, the interests of the regime, not the people of China, are paramount and their ,main strategic direction' (supreme objective) is to take Taiwan, ending its life as a de facto independent country. “23 Diesem obersten Ziel steht bisher vor allem die Schutzmachtrolle der USA im Wege.

Doch nicht nur gegenüber der „abtrünnigen Provinz“ Taiwan wendet die Volksrepublik zunehmend aggressive Methoden an. Im Ost- und Südchinesischen Meer nutzt der chinesische Staat hybride Methoden und paramilitärische Kräfte, um Gebietsansprüche gegen schwächere Anrainer auch unter bewusster Missachtung internationalen Rechts durchzusetzen. Indien war erst kürzlich in einen gewaltsamen Grenzkonflikt mit China verwickelt. Mög-

22 Zur Situation der Jahre 2016/17 s. Ministry of National Defense, R.O.C. (2017): National Defense Report, https://www.mnd.gov.tw/ NewUpload/歷年國防報告書網頁專區/歷年國防報告書專區.files/ 國防報告書-106/國防報告書-106-英文版.pdf, 44; zur jüngeren Entwicklung s. Eric Cheung/Brad Lendon: China sends more than three dozen warplanes in skies near Taiwan in largest incursion yet, $C N N$, 2.10.2021, https://edition.cnn.com/2021/10/01/asia/chinataiwan-warplane-incursion-record-intl-hnk-ml/index.html; Taiwan's Airspace Sees Increase In Chinese Military Incursions, Statista, 14.4.2021, https://www.statista.com/chart/24620/chinese-militaryaircraft-entering-taiwans-adiz/.

23 Easton 2017, 16.

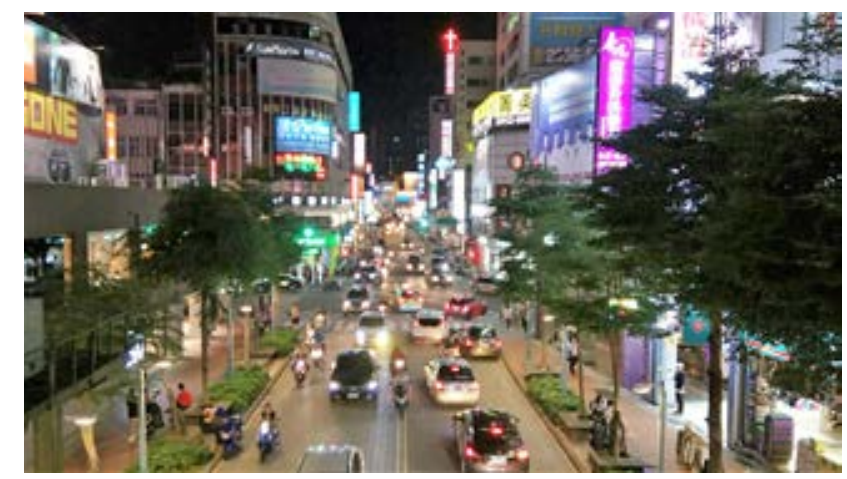

Taipeh, die Hauptstadt Taiwans bei Nacht

licherweise inspiriert durch Russland,${ }^{24}$ wendet China auch gegen weitere Nachbarn vielfältige weitere hybride Formen der Einflussnahme und der Auseinandersetzung an. Das Spektrum reicht von Geiseldiplomatie, erpresserischem Ausnutzen von Handelsabhängigkeiten (besonders sichtbar im Zusammenhang mit der Verknappung medizinischer Produkte zu Beginn der COVID-Pandemie und im Handelsstreit mit Australien sowie durch Versuche, die Beschaffung von Impfstoffen durch Taiwan zu verhindern), rechtlicher Kriegführung (lawfare) gegen Kritiker Chinas; sogenannter „Wolfskrieger-Diplomatie“, massiver Informationskriegsführung und Desinformation in den Medien bis hin zu „autoritärer Einflussnahme“, mit deren Hilfe missliebige Individuen in anderen Ländern angegriffen oder Unterstützernetzwerke kultiviert werden. ${ }^{25}$

\section{Totalitäre Wende bei massiver Aufrüstung: Bereitet sich China auf einen Konflikt vor?}

Sorge sollte NATO-Beobachtern in diesem Zusammenhang die aktuelle innenpolitische Entwicklung Chinas machen. Dort hat sich seit dem Amtsantritt Xi Jinpings 2012/13 ein beunruhigender innenpolitischer Umschwung vollzogen, der Konsequenzen auch für den Rest der Welt hat. Im Inland muss die zunehmende Machtkonzentration um den KP-Generalsekretär Xi und der Aufbau eines totalitären Überwachungsstaates nicht zuletzt aufgrund der gravierenden damit verbundenen Menschenrechtsverletzungen beunruhigen. ${ }^{26}$ Und während westliche Staaten

\section{Goldstein 2015.}

25 Speranza 2020.

26 Strittmatter 2018; Ben Westcott: Xi Jinping set out to save the Communist Party. But critics say he made himself its biggest threat, 
noch darüber debattieren, wie ein „Kalter Krieg 2.0“ und eine Entkoppelung von Wertschöpfungsketten tunlichst vermieden werden können, beschränkt China bereits massiv die Freiheiten des heimischen Wirtschaftssektors, strebt technologische Autarkie an und erschwert unter dem Vorwand der Pandemiebekämpfung zusehends den rein zivilgesellschaftlichen Austausch mit dem Ausland. ${ }^{27}$

Dieser „Große Sprung nach Links“ geht mit einem deutlich aggressiveren Verhalten Chinas in der Außenund Sicherheitspolitik einher. Diese Entwicklung ist von rüstungspolitischen Maßnahmen begleitet, die das Ziel haben, Chinas Streitkräfte bis zum 100-jährigen Staatsjubiläum 2049 zu den schlagkräftigsten der Welt zu machen. Eine historisch für die Nachkriegsära beispiellose Flottenrüstung, die Chinas Marine in kürzester Zeit durch mehrere Flugzeugträger-Kampfgruppen und eine neue Flotte großer amphibischer Angriffsschiffe eine offensive Machtprojektionsfähigkeit ermöglichen soll, ist in vollem Gange. Die Geschwindigkeit dieser Aufrüstung ist für Friedenszeiten absolut untypisch und nicht durch Ersatzbeschaffung für obsolete Einheiten zu erklären. So wurde allein zwischen 2013 und 2021, also innerhalb von nur acht Jahren, eine völlig neue Schiffsklasse von 72 Korvetten in Dienst gestellt. Die Tonnage aller zwischen 2014 und April 2018, also innerhalb von weniger als fünf Jahren von der VBA-Marine neu in Dienst gestellten Marineschiffe entsprach laut einer Untersuchung des IISS der Gesamttonnage der britischen Royal Navy, Europas größter Marine, bzw. der Gesamttonnage der japanischen Marine (der nach China größten Marine Ostasiens). ${ }^{28}$ Die Gesamtzahl aller chinesischen Marineschiffe übersteigt bereits jetzt die der US Navy, auch wenn deren Schiffe teils größer und im Schnitt auch besser bewaffnet sind. Allerdings sind die Schiffe der US Navy weltweit im Einsatz, während sich die chinesische Marine weitgehend auf Ostasien konzentrieren kann und einen gewaltigen Heimvorteil genießt, darunter eine Luftabdeckung vom eigenen Festland aus. Im Südchinesischen Meer entsteht zur Zeit mit Hilfe der von China militarisierten künstlichen Inseln eine Seeraum-Überwachungsinfrastruktur, das Blue Ocean Information Network, das durch Zusammenschluss einer Vielzahl von statischen und mobilen Sensoren und unter Verwendung von Zukunftstechnologien und Weltrauminfrastrukturen sogar in der opaken Unterwasserdimension für Transparenz und Kon-

CNN, 26.7.2021, https://edition.cnn.com/2021/07/25/china/xi-jinpingccp-100-party-intl-hnk/index.html. https://www.nato.int/cps/en/ natohq/news_171458.htm.

27 David Ignatius: Opinion: Xi Jinping's disturbing Maoist turn, The Washington Post, 21.9.2021.

28 Childs/Waldwyn 2018. trolle sorgen soll. ${ }^{29}$ Beobachter gehen davon aus, dass dies der Absicherung eines geplanten strategischen U-BootSanktuariums dient, um eine sichere Zweitschlagsfähigkeit aufzubauen. ${ }^{30}$

Auffällig ist insbesondere der für Friedenszeiten äußerst ungewöhnliche, beschleunigte Baurhythmus chinesischer Marineschiffbauprogramme: Chinas Werften bauen demnach Kriegsschiffe rund um die Uhr, an drei Achtstunden-Schichten pro Tag und an sieben Tagen pro Woche. Die einzelnen Beschaffungsvorhaben sind für die Außenwelt jeweils intransparent und werden stets erst verzögert und nach dem Vorliegen von unabhängigen Beweisen in Form etwa von Satellitenfotos öffentlich kommuniziert - wenn überhaupt. ${ }^{31}$ Es besteht zur Zeit in offenen Quellen zum Beispiel Unklarheit bezüglich der bereits existierenden und der geplanten Anzahl nukleargetriebener U-Boote, insbesondere der strategischen Boote.

Auch die Anzahl der von China angestrebten Trägerkampfgruppen ist noch immer unklar. Inoffizielle Äußerungen chinesischer Militärvertreter deuten jedoch darauf hin, dass „mindestens sechs“ geplant sein könnten, für die wiederum neben dem Stützpunkt in Dschibouti „mindestens zehn Marinestützpunkte in Übersee“ zu schaffen wären, idealerweise auf allen Kontinenten. ${ }^{32}$ Auffällig ist ebenfalls die hohe Anzahl großer amphibischer Angriffsschiffe, die als Träger für senkrechtstartende Flugzeuge, Hubschrauber und Drohnen dienen, die von China derzeit beschafft werden. Acht Einheiten des Typs 071 und einer des deutlich größeren Typs 075 sind bereits in Dienst gestellt, mindestens weitere sieben des letzteren Typs befinden sich im Bau. ${ }^{33}$ Analog zur Marinerüstung findet auch eine massive Modernisierung der Luftwaffe statt. Eine Militärreform hat zudem die organisatorischen Voraussetzungen dafür geschaffen, die Streitkräfte insgesamt schlagkräftiger $\mathrm{zu}$ machen und besonders ihre asymmetrischen „Systemzerstörungs“-Kapazitäten und Weltraumfähigkeiten in einer neuen Teilstreitkraft, der Strategischen Kampfunterstützungstruppe, zu bündeln.

29 AMTI 2020.

30 Vgl. Kirchberger/O’Keeffe 2019.

31 Andrew Tate: China Quietly Increasing Warship Numbers, Jane's Defence Weekly, 21.9.2018; Vinayak Bhat: High-speed production: Chinese navy built 83 ships in just eight years, The Print, 20.9.2017, https:// theprint.in/defence/chinese-navy-built-83-ships-8-years/10416/. 32 Xin Liu/Caiyu Liu: China Eyes Building More Aircraft Carriers, Global Times, 20.4.2017, http://www.globaltimes.cn/content/1043455. shtml.

33 Yasmin Tadjdeh: China Building Formidable Amphibious Fleet, National Defense, 25.6.2021, https://www.nationaldefensemagazine. org/articles/2021/6/25/china-building-formidable-amphibious-fleet. 


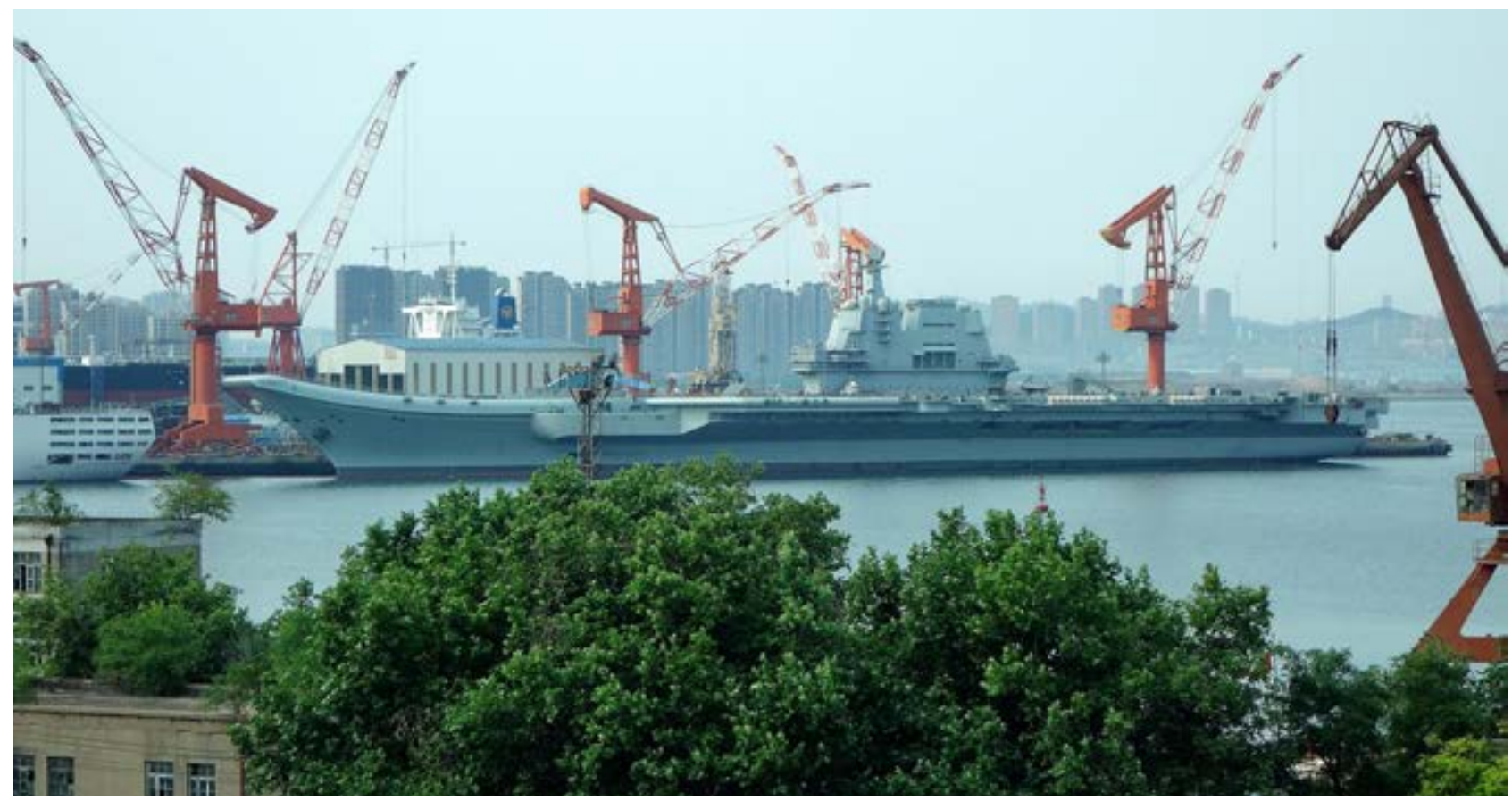

Foto des ersten vollständig in China gebauten Flugzeugträgers Shandong in der Werft

Zugleich erfolgt eine rasante Modernisierung des nuklearen Arsenals mit dem Ziel, eine vollständige nukleare Triade aufzubauen. Drei neue Raketensilo-Anlagen, die kürzlich auf Satellitenbildern in Westchina entdeckt wurden, deuten auf eine geplante massive Ausweitung des Arsenals an landgestützten Raketen hin. ${ }^{34}$ Außerdem betreibt China intensiv die Forschung und Entwicklung von Zukunftstechnologien in der Waffenentwicklung, darunter Hyperschallwaffen, militärische Quantentechnologien sowie unbemannte Systeme inklusive Schwarm-Technologie. China ist mittlerweile führend auf einigen Gebieten, etwa bei militärischen Anwendungen von Künstlicher Intelligenz. ${ }^{35}$

Insgesamt scheint es, dass China unter Einsatz enormer Finanzmittel versucht, eine überwältigende konventionelle Überlegenheit im ostasiatischen Raum herzustellen und dabei genügend Redundanz für den Krisenfall aufzubauen und zugleich nukleare „strategische Stabilität" mit den USA zu erreichen. ${ }^{36}$ Letztere soll verhindern,

34 Die Entdeckung der drei neuen Raketensilos wurde separat dokumentiert in Kristensen 2021, Korda/Kristensen 2021 und Bill Gertz: Exclusive: China Building Third Missile Field for Hundreds of New ICBMs, The Washington Times, 12.8.2021. Für eine Einschätzung der Bedeutung s. Davis 2021.

35 Kirchberger/Mohr 2020, 59-63.

36 Mathieu Duchâtel: How China is relying on Russia to achieve 'strategic stability' with the US, South China Morning Post, 13.10.2019, dass in einem regionalen, von China begonnenen Krieg gegen einen Verbündeten Washingtons, die USA auf eine Form der erweiterten nuklearen Abschreckung zurückgreifen. Dabei versucht Beijing, größtmöglichen Nutzen aus den Möglichkeiten zu ziehen, die sich dem Militär durch verschiedene Zukunftstechnologien der „Vierten Industriellen Revolution" bieten.

Im Angesicht dieser Entwicklungen folgte die vielfach kritisierte Entscheidung Präsident Bidens, den unter Trump beschlossenen Abzug aus Afghanistan trotz internationaler Kritik und großer Reibungsverluste umzusetzen, der Logik, sich auf die aus US-Sicht existenzielle Herausforderung durch China zu konzentrieren. Vor diesem Hintergrund greifen Sichtweisen, die die Interessen der europäischen NATO-Staaten und der NATO insgesamt durch Chinas Aufstieg höchstens indirekt oder mittelbar berührt sehen, deutlich zu kurz.

Aus chinesischer Sicht wiederum ist ein Zustand der Uneinigkeit in der NATO über die Natur der chinesischen Herausforderung wünschenswert: $\mathrm{Zu}$ verhindern, dass die NATO-Partner zu einer gemeinsamen Lage-Einschätzung kommen können, kann sogar als ein zentrales Ziel chinesischer Einflussnahme auf europäische Länder angenommen werden. Umgekehrt signalisiert die scharfe Reaktion

https://www.scmp.com/news/china/diplomacy/article/3032436/ how-china-relying-russia-achieve-strategic-stability-us. 
aus Beijing auf die Erwähnung Chinas als „Herausforderung“ im Abschluss-Communiqué des NATO-Gipfels vom Juni 2021, dass die beginnende Umorientierung der NATO dort nicht nur zur Kenntnis genommen wurde, sondern durchaus Besorgnis auslöst. ${ }^{37}$

\section{Welche Rolle könnte die NATO bei der Eskalationsvermeidung spielen?}

Beijing droht dem demokratisch regierten und de facto unabhängigen Taiwan unverhohlen mit Krieg, wenn es sich einer Vereinigung widersetzt, und rüstet in einem Tempo und Ausmaß auf, das für Friedenszeiten äußerst untypisch ist. All dies geschieht vor dem Hintergrund einer sich stetig vertiefenden Militär- und Sicherheitskooperation mit Russland, die inzwischen nicht nur Waffenexporte der modernsten russischen Systeme umfasst, sondern auch die gemeinsame Entwicklung von Waffensystemen sowie die Hilfe Russlands bei strategischen Systemen wie bei dem Aufbau eines Frühwarnsystems für ballistische Raketenangriffe und die Zusammenarbeit bei ballistischer Raketenabwehr. ${ }^{38}$ Hier zeichnet sich die Möglichkeit ab, dass eine eurasische Achse Russland-China entsteht, die Rückwirkungen bis nach Europa entfalten würde.

Die NATO sollte sich angesichts der sich aktuell stetig verschlechternden Beziehungen zwischen China und den USA insbesondere drei Fragen stellen:

1) Falls der Worst Case eines heißen Konflikts im Westpazifik eintritt, mit allen Risiken einer Eskalation zum großen Krieg, die damit verbunden wären: Welche unmittelbaren Folgen hätte dies für die NATO-Staaten, und wie positioniert sich die NATO hierzu? Für ihre Einschätzung sollte die NATO das vorhandene Wissen der NATO-Partnerländer in der Asien-Pazifik-Region nutzen und deren Risikoeinschätzungen ernst nehmen.

2) Was wäre, falls Russland eine solche Situation zu seinem Vorteil ausnutzt und an der Peripherie Europas zeitgleich eine Eskalation provoziert? Wie kann dem vorgebeugt werden, und wären die europäischen NATO-Mitglieder in der Lage, auch mit verringertem Engagement Amerikas dem entgegenzutreten?

37 Steven Erlanger/Michael D. Shear: Shifting Focus, NATO Views China as a Global Security Challenge, The New York Times, 14.6.2021 online, updated 30.9.2021.

38 Korolev 2020.
3) Wie kann die NATO aktiv ihren Teil dazu beitragen, dass es zu einer kriegerischen Eskalation im Westpazifik gar nicht erst kommt?

Es ist im ureigenen Interesse aller Mitglieder der transatlantischen Allianz, zu verhindern, dass infolge kollektiven Schlafwandelns ein verheerender Konflikt ausbricht, der durch wirksame Abschreckung hätte vermieden werden können.

Hierzu sollte die NATO sich ihrer eigenen Einflussmöglichkeiten auf die Lage im Westpazifik stärker bewusst werden und diese auch nutzen. Das bisherige taktische Vorgehen Chinas zur Schaffung eines neuen Status quo in seiner Peripherie beruht nicht unwesentlich auf einer Verschleierung eigener Aktivitäten und zahlenmäßiger Überrumpelung überforderter Gegenparts. Verstärkte öffentliche Aufmerksamkeit durch systematisches Monitoring der NATO und entschiedene verbale Reaktionen von Seiten der NATO und ihrer Mitglieder zur Unterstützung schwächerer Anrainerstaaten allein haben bereits das Potenzial, die Effektivität einiger dieser hybriden Taktiken zumindest zu verringern. Vorbeugend sollte die NATO daher ein noch deutlicheres Interesse als bisher an einer systematischen Überwachung der gefährlichen Hot Spots und am institutionalisierten Datenaustausch mit NATO-Partnerstaaten im Westpazifik signalisieren, um im Verbund mit den Anrainerstaaten der bisher so erfolgreichen chinesischen Salamitaktik entgegenzuwirken.

Die bereits von den NATO-Mitgliedern Großbritannien und Frankreich praktizierte militärische Präsenz hilft den USA dabei, eine wirkungsvolle Abschreckungskulisse aufzubauen. Weitere NATO-Staaten könnten sich je nach Fähigkeiten und Kapazitäten daran von Zeit zu Zeit auf freiwilliger Basis beteiligen. Ideal wäre eine kontinuierliche, in Arbeitsteilung zu realisierende europäische Präsenz.

Währenddessen ist eine Gegenmachtbildung im Pazifik bereits in vollem Gange, mit AUKUS als ihrem bisher eindrucksvollsten Symbol. Doch auch Indien, Vietnam, Japan, die Philippinen und Südkorea haben in vielfältiger Weise, nicht zuletzt durch eigene große Rüstungsprojekte, auf die neuen Herausforderungen durch China reagiert und orientieren sich sämtlich stärker an den USA. Diese für China nachteilige Entwicklung hat dort bereits unter inländischen Beobachtern Besorgnis ausgelöst, dass genau der Effekt einzutreten scheint, vor dem schon Deng Xiaoping für den Fall eines zu früh auftrumpfenden Chinas gewarnt und zu vermeiden gesucht hatte. ${ }^{39}$

39 Ben Westcott: Xi Jinping set out to save the Communist Party. But critics say he made himself its biggest threat, CNN, 26.7.2021, https:// 
Die NATO als großes Bündnis westlicher Demokratien genießt auch in Asien diplomatisch ein hohes Gewicht. Ihre 30 Mitgliedstaaten stellen, wie Generalsekretär Stoltenberg im November 2019 in Erinnerung rief, zusammen etwa die Hälfte der globalen Wirtschafts- und Militärmacht. ${ }^{40}$ Ihre Positionierung beeinflusst somit unmittelbar die Risikokalkulation Beijings. Ein Angriff Chinas auf einen schwächeren Anrainer, der unter dem Schutz der USA steht, wird umso wahrscheinlicher, je mehr Beijing das Risiko einer geschlossenen Front als gering bewertet und zudem vermuten darf, dass die USA ihre Schutzmachtrolle nicht wirklich ausfüllen würden. Er wird dagegen umso unwahrscheinlicher, je mehr Beijing eine reelle Gefahr sieht, einen Konflikt mit den USA führen zu müssen, wenn diese auch noch von der NATO unterstützt würden, mit allen negativen diplomatischen und wirtschaftlichen Folgen, die das für China hätte. Versuche Beijings, die NATO zu spalten und Kräfte in der NATO zu stärken, die für „Neutralität“ gegenüber China plädieren, werden somit unweigerlich zunehmen. Die NATO-Mitgliedstaaten sollten dies nicht zulassen, sondern sollten klarstellen, dass die Loyalität der NATO mit ihrem wichtigsten Mitgliedstaat im Fall eines chinesischen Angriffs gegen US-Kräfte im Westpazifik nicht in Frage steht. Zugleich könnte die NATO China in Form eines neu zu schaffenden NATO-China-Rats analog zum NATO-Russland-Rat ein institutionalisiertes Dialog-Angebot unterbreiten. $\mathrm{Ob}$ China davon letztlich Gebrauch machen würde, stünde zwar abzuwarten. Dennoch könnte ein solcher Mechanismus ein wichtiger Teil einer kohärenten China-Strategie werden, die auf Abschreckung und Dialog zugleich setzt. ${ }^{41}$

Letztlich muss aber die Verteidigung Europas zur Hauptaufgabe der europäischen NATO-Mitgliedsstaaten werden, die in Zukunft in der Lage sein müssen, im schlimmsten Fall einer gleichzeitigen Krise in Ostasien und in Europa auch mit verringerter amerikanischer Unterstützung eine wirkungsvolle Abschreckung gegenüber Russland aufrecht zu erhalten.

edition.cnn.com/2021/07/25/china/xi-jinping-ccp-100-party-intl-hnk/ index.html.

40 NATO Secretary General announces increased defence spending by Allies, NATO, 29.11.2019, https://www.nato.int/cps/en/natohq/ news_171458.htm.

41 Siehe die detaillierteren Vorschläge hierzu in Binnendijk/Kirchberger 2021, 5; 87-93.

\section{Literaturverzeichnis}

AMTI (2020): Exploring China's Unmanned Ocean Network, CSIS Asia Maritime Transparency Initiative, 16.6.2020, https://amti. csis.org/exploring-chinas-unmanned-ocean-network/

Binnendijk, Hans/Kirchberger, Sarah (2021): The China Plan: A Transatlantic Blueprint for Strategic Competition. Washington DC: Atlantic Council

Blasko, Dennis J. (2009): China's Views on NATO Expansion: A Secondary National Interest, Jamestown Foundation China Brief, Vol. 9 Issue 5, 4.3.2009

Brzezinski, Zbigniew (1997): The Grand Chessboard: American Primacy and Its Geostrategic Imperatives. New York: Basic Books

Childs, Nick/Waldwyn, Tom (2018): China's Naval Shipbuilding: Delivering on Its Ambition in a Big Way, Military Balance Blog, International Institute for Strategic Studies, 1.5.2018, https:// www.iiss.org/blogs/military-balance/2018/05/china-navalshipbuilding.

Cole, J. Michael (2019): The Principal Targets of CCP’s ‘Sharp Power' Operations Against Taiwan, The Global Taiwan Brief 4(22), 20.11.2019, 7-9, https://globaltaiwan.org/2019/11/ vol-4-issue-22/

Cooley, Alexander/Nexon, Daniel (2020): Exit from Hegemony: The Unraveling of the American Global Order. Oxford: Oxford University Press

Davis, Malcolm (2021): China Military Watch, The Strategist, Australian Strategic Policy Institute (ASPI), August 20, 2021, https://www.aspistrategist.org.au/china-military-watch-10/

Dreyer, June Teufel (2011): People's Liberation Army Lessons from Foreign Conflicts: The Air War in Kosovo, in: Andrew Scobell/ David Lai/Roy Kamphausen (Hrsg.): Chinese Lessons from Other People's Wars. Carlisle, PA: US Army War College Strategic Studies Institute, 33-73

Dreyer, June Teufel (2018): The Big Squeeze: China’s Anaconda Strategy to Force Taiwan to Surrender, Foreign Policy Research Institute e-Notes, 13.8.2018, https://www.fpri.org/ article/2018/08/the-big-squeeze-beijings-anaconda-strategyto-force-taiwan-to-surrender/

Easton, Ian (2017): The Taiwan Invasion Threat: Taiwan's Defense and American Strategy in Asia. Arlington, VA: Project 2049 Institute

Engstrom, Jeffrey (2018): Systems Confrontation and System Destruction Warfare: How the Chinese People's Liberation Army Seeks to Wage Modern Warfare. Santa Monica, CA: RAND Corporation

Goldstein, Lyle J. (2015): Get Ready: China Could Pull a 'Crimea' in Asia, The National Interest, 11.4.2015, https:// nationalinterest.org/feature/get-ready-will-china-pullcrimea-asia-12605.

Holslag, Jonathan (2015): Frieden auf Chinesisch: Warum in Ostasien Krieg droht. Hamburg: edition Körber-Stiftung

Kim, Jina (2020): China and Regional Security Dynamics on the Korean Peninsula. Washington, D.C.: Carnegie Endowment for International Peace

Kirchberger, Sarah/Mohr, Johannes (2020): China's Defence Industry, in: Keith Hartley/Jean Bélin (Hrsg.): The Economics of the Global Defence Industry. London: Routledge, 35-68

Kirchberger, Sarah/O'Keeffe, Patrick (2019): Chinas schleichende Annexion im Südchinesischen Meer - die strategischen 
Hintergründe, Sirius - Zeitschrift für strategische Analysen, $3(1), 3-20$

Korda, Matt/Kristensen, Hans (2021): China Is Building A Second Nuclear Missile Silo Field, Strategic Security Blog, Federation of American Scientists, 26.7.2021, https://fas.org/blogs/ security/2021/07/china-is-building-a-second-nuclear-missilesilo-field/

Korolev, Alexander (2020): China-Russia cooperation on missile attack early warning systems, East Asia Forum, 20.11.2020, https://www.eastasiaforum.org/2020/11/20/china-russiacooperation-on-missile-attack-early-warning-systems/

Kristensen, Hans M. (2021): China's Expanding Missile Training Area: More Silos, Tunnels, and Support Facilities, Strategic Security Blog, Federation of American Scientists, 24.2.2021, https://fas.org/blogs/security/2021/02/plarf-jilantaiexpansion

Liu, Xinhua刘新华 (2015): Zhongguo fazhan haiquan zhanlüe yanjiu (A Study on China's Strategy of Development of Sea Power). Peking: Renmin chubanshe
Qiao, Liang / Wang, Xiangsui (2015): Unrestricted Warfare: Translated from the Original People's Liberation Army Documents (die chin. Originalpublikation erschien 1999). Brattleboro, VT: Echo Point Books \& Media, https://www.c4i. org/unrestricted.pdf

Roberts, Brad (2020): Neue Herausforderungen erfordern neue Ideen. Elemente einer Theorie des Sieges in modernen strategischen Konflikten, Sirius - Zeitschrift für strategische Analysen, 4 (4), 410-434

Speranza, Lauren (2020): A Strategic Concept for Countering Russian and Chinese Hybrid Threats. Washington DC: Atlantic Council, Juli 2020

Strittmatter, Kai (2018): Die Neuerfindung der Diktatur: Wie China den digitalen Überwachungsstaat aufbaut. München: Piper

Zhang, Wenmu张文木 (2014): Ukelan shiji de shijie yiyi jiqi dui Zhongguo de jingshi (The Ukraine Crisis: What does it Mean to the World and China), in Guoji anquan yanjiu (Journal of International Security Studies) 2014 (4), 1-26 